REVISTA MATEMÁTICA de la

Universidad Complutense de Madrid

Volumen 10, número 1: 1997

http://dx.doi.org/10.5209/rev_REMA.1997.v10.n1.17507

\title{
Rational models of solvmanifolds with Kählerian structures.
}

\author{
A. TRALLE (SZCZECIN)
}

\begin{abstract}
We investigate the existence of symplectic non-Kählerian structures on compact solvmanifolds and prove some results which give strong necessary conditions for the existence of Kählerian structures in terms of rational homotopy theory. Our results explain known examples and generalize the Benson-Gordon theorem [BG2] (our method allows us to drop the assumption of the complete solvability of $G$ ).
\end{abstract}

\section{Introduction}

There has been an interest recently in examples of compact symplectic manifolds with no Kähler structures ([Ab, Gmf, CFG, FLS, McD, $\mathrm{LuO}]$ and others). With the exception of [McD, FLS] and the surgery technique of [Gmf], known examples are nilmanifolds coming from the following general theorem proved by Benson and Gordon [BG1] and Hasegawa [Has].

Let $M$ be a compact $K(\Gamma, 1)$-manifold where $\Gamma$ is a discrete, finitely generated, torsion free, nilpotent group. If $M$ admits a Kähler structure, then $\Gamma$ is abelian and $M$ has the homotopy type of a torus.

This theorem implies that any non-toral symplectic $K(\Gamma, 1)-$ manifold with nilpotent $\boldsymbol{\Gamma}$ yields the desired example.

Nevertheless, it would be interesting to look for other aspherical

1980 Mathematics Subject Classification: (1985 Revision). 53C15, 55P62.

Servicio Publicaciones Univ. Complutense. Madrid, 1997. 
manifolds which are symplectic but non-Kählerian. For example, the result of [FLS] suggests looking for such manifolds in the class of solvmanifolds. Observe once more that the fundamental group of a solvmanifold need not be nilpotent, (moreover, it is known that for a solvmanifold $M$, the nilpotency of $\pi_{1}(M)$ implies the diffeomorphicity of $M$ to a nilmanifold [VGS]).

The case of solvmanifolds, however, differs essentially from that of the nilmanifolds for several reasons. In general, the Nomizu theorem concerning the cohomology of a nilmanifold is not available, therefore, the minimal model of a solvmanifold cannot be used directly. Moreover, the known examples of symplectic and Kählerian solvmanifolds show that all possible homotopy types, Kählerian and non-Kählerian, may oecur (see [FLS, BG2]). The most unexpected example of [BG2] shows that there exists a compact symplectic solvmanifold of the same rational homotopy type as $T^{2} \times S^{2}$, but non-Kählerian, because it cannot carry any complex structure $[\mathrm{FG}]$.

In [BG2] and [FLS], the authors investigated the case of a solvmanifold $G / \Gamma$ of a completely solvable Lie group $G$ and established the necessary conditions for the existence of Kählerian structure on such manifolds. The key to the proof of the cited results is the NomizuHattori theorem $[\mathrm{H}]$ regarding the cohomology of $G / \Gamma$ which allowed the authors to establish the rational model $\left(\bar{A}^{*}(G / \Gamma), d\right)=\left(\Lambda L(G)^{*}, \delta\right)$. In general, the Nomizu-Hattori theorem does not hold (see [R] and [VGS]) and the technique of [BG2] is not available. Moreover, there are many types of solvmanifolds which are not completely solvable [VGS], e.g. solvmanifolds of $(R)$-type, $(E)$-type, mixed types etc.

Nevertheless, the main result of this paper shows that there exists a graded differential algebra $\left(A^{*}(G / \Gamma), d\right)$ which is a rational model of an arbitrary Kählerian solvmanifold and possesses the same algebraic properties as $\left(\bar{A}^{*}(G / \Gamma), d\right)$. Of course, in the completely solvable case these algebraic properties determine the structure of $G$ (it is semidirect product of abelian and nilpotent parts). We use a different approach based on the Thomas theorem [Th1], [Th2] which describes models of Serre fibrations. The use of this theorems is of independent interest.

To formulate the main result of the paper, we introduce the notion of the twisted tensor product of graded commutative differential algebras. Here and in the sequel we use traditional notations of rational homotopy 
theory and solvmanifolds. Nevertheless, some of them are explained in the next section.

Let $(\mathcal{R}, \bar{d})$ and $(\mathcal{S}, \delta)$ be graded commutative differential algebras. Introduce the tensor product $\mathcal{R} \otimes S$ of $\mathcal{R}$ and $\mathcal{S}$ as graded algebras and define the "twisted" derivation $d$ by the formula

$$
\begin{gathered}
d(r \otimes 1)=\vec{d}(r) \otimes 1, r \in \mathcal{R} \\
d(1 \otimes s)=1 \otimes \delta(s)+\sum_{i \geq 0}(-1)^{(i+1) \operatorname{deg}(s)} \sum_{\xi \geq 1} \phi_{i}^{\xi}(s) \otimes r_{i+1}^{\xi}, s \in \mathcal{S}
\end{gathered}
$$

here $\phi_{i}^{\xi}$ is a derivation of $(\mathcal{S}, \delta)$ decreasing the degree by $i$, that is

$$
\phi_{i}^{\xi}(x y)=\phi_{i}^{\xi}(x) y+(-1)^{i \cdot \operatorname{deg}(x)} x \phi_{i}^{\xi}(y)
$$

and $\left\{r_{i+1}^{\xi}, \xi=1,2, \cdots\right\}$ constitute a basis of $\mathcal{R}^{i+1}$. By definition, the graded differential algebra $(\mathcal{R} \otimes \mathcal{S}, d)$ is called the twisted tensor product of $(R, \bar{d})$ and $(\mathcal{S}, \delta)$. In the sequel we denote the twisted tensor product by

$$
(\mathcal{R}, d) \otimes_{\tau}(\mathcal{S}, \delta)
$$

Theorem. Let $M=G / \Gamma$ be a compact solvmanifold carrying a Kählerian structure. There exists a free graded differential algebra $\left(A^{*}(E), d\right)$ satisfying the following properties

(i) $\left(A^{*}(E), d\right)$ is a Lefschetz algebra and a rational moder for $E$;

(ii) $\left(A^{*}(E), d\right)=(\Lambda X, \bar{d}=0) \otimes_{\tau}(\Lambda Y, \delta), X$ and $Y$ are finite dimensional vector spaces of elements of degree 1 , and $(\Lambda Y, \delta)$ is a minimal differential algebra;

(iii) for the bigrading $\Lambda^{i j}=\Lambda^{i} X \otimes \Lambda^{j} Y$ the equalities hold:

$$
H^{1}\left(A^{*}\right)=\Lambda^{1,0}, H^{n-1}\left(A^{*}\right)=\Lambda^{k, l-1}
$$

(iv) the Lefschetz element $\omega \in \Lambda^{2}(X \oplus Y)$ can be chosen in the form

$$
\omega=\omega^{2,0}+\omega^{0,2}
$$

and $\omega^{2,0}$ and $\omega^{0,2}$ are non-degenerate 2-forms on $X^{*}$ and $Y^{*}$, which are closed and non-exact with respect to $d$ and $\delta$. 
(v) $\operatorname{dim} X$ and $\operatorname{dim} Y$ are even and $X^{*}$ and $Y^{*}$ are $\omega-$ orthogonal.

To illustrate the usefulness of theorem 3 we also show that it generalizes the Benson-Gordon theorem [BG2]. Here and in the sequel $L(G)$ denotes the Lie algebra of the Lie group $G$.

Corollary. If $G$ is completely solvable and $G / \Gamma$ is a solvmanifold that admits a Kahler structure, then

(i) there is an abelian complement $\mathcal{A}$ in $L(G)$ of the derived algebra $\mathcal{N}=[L(G), L(G)]$

(ii) $\mathcal{A}$ and $\mathcal{N}$ are even dimensional;

(iii) the center of $L(G)$ intersects $\mathcal{N}$ trivially;

(iv) the Kahlerian form is cohomologous to a left invariant sympletic form $\omega=\omega_{0}+\omega_{1}$, where $\mathcal{N}=\operatorname{ker}\left(\omega_{0}\right)$ and $\mathcal{A}=\operatorname{ker}\left(\omega_{1}\right)$;

(v) both $\omega_{0}$ and $\omega_{1}$ are closed but not exact in $L(G)$ and also in $\mathcal{N}$ and $\mathcal{A}$;

(vi) the adjoint action of $\mathcal{A}$ on $\mathcal{N}$ is by infinitesimal symplectomorphisms.

\section{Remark.}

(i) Corollary follows rather from the proof of Theorem 3.

(ii) The properties (ii)-(v) stated in the Theorem are the algebraic properties established in [BG2] for $\left(\Lambda L(G)^{*}, \delta\right)$ in the completely solvable cáse.

In the last section of the paper we construct explicitly an example of a sympletic solvmanifold which is not completely solvable. We deduce that this solvmanifold is not Kählerian. We use the algebraic properties of the model $\left(A^{*}(G / \Gamma), d\right)$ established in the Theorem for Kählerian solvmanifolds to describe the rational homotopy type of any 4-dimensional Kählerian solvmanifold. This description explains examples in [FG] and [BG2] from the rational homotopy point of view. 


\section{Preliminaries}

This work is devoted, in fact, to algebraic properties of free models of Kählerian compact solvmanifolds $G / \Gamma$, that is free graded differential algebras $A^{*}(G / \Gamma)$, quasiisomorphic to the Rham algebra $\Omega_{D R}(G / \Gamma)$. Thus, we are in the framework of rational homotopy theory, although our models are not minimal in general. Since there are many books and research articles on rational homotopy theory, we assume the reader to be familiar with it, referring to [Ha], [Le], [Ta] and [DGMS].

In the sequel we consider the category $R-D G A$ of graded differential algebras over the field of the real numbers (although the algebraic results are valid for the arbitrary field of a zero characteristic). By definition, a model of a manifold $M$ is a free graded differential algebra $A^{*}(M)$ such that there exists either a homomorphism,

$$
\alpha: A^{*}(M) \rightarrow \Omega_{D R}(M)
$$

or a homomorphism

$$
\beta: \Omega_{D R}(M) \rightarrow A^{*}(M)
$$

inducing isomorphism in cohomology. Of course, $A^{*}(M)$ need not be minimal and therefore is not unique. We use the notion of the minimal model in the usual sense. The cohomology functor for $R-D G A$ is denoted by $H^{*}$. By definition we call a free finitely-graded differential algebra

$$
A=\oplus_{i=0}^{n} A^{i}
$$

oriented if $H^{n}(A) \neq 0$.

\section{Definition.}

(i) A finitely-graded free differential algebra $(A, d)=\left(\oplus_{i=0}^{2 n} A^{i}, d\right)$ is called symplectic, if there exists an element $[\omega] \in H^{2}$, such that $|\omega|^{n} \neq 0, \omega \in A^{2}$;

(ii) An algebra $(A, d)$ satisfying (i) is called Lefschetz, or satisfies the hand Lefschet $z$ condition if all homomorphisms

$$
L_{\omega^{r}}: H^{n-r} \rightarrow H^{n+r}, L_{\omega^{r}}([h])=\left[\omega^{r}\right][h], r \geq 1
$$

ane isomorphisms. The element $\omega \in A^{2}$ is called Lefschetz. 
Observe that symplectic algebras satisfying Poincaré duality are models of compact symplectic manifolds and Lefschetz algebras are those of compact Kählerian manifolds. This fact will play the key role in Section 3.

One of the main ingredients of proofs in this article is the Thomas theorem concerning the models of rational Serre fibrations [Th 1] and [Th 2], therefore, we reproduce its exact formulation (see [Th 1] for the proof).

Let

$$
F \rightarrow E \rightarrow B
$$

be a Serre fibration with $F$ and $B$ of finite cohomology type. It is a known fact that the fundamental group $\pi_{1}(B)$ acts naturally on $H^{j}(F), j \geq 1$, that is, there exists a representation of $\pi_{1}(B)$ in $A u t\left(H^{*}(F)\right)$ (see, e.g. [Se]). We presume that, $E, F, B$ are path connected.

Recall that by definition an action of a group $G$ on an abelian group' $C$ is called nilpotent if the following conditions are satisfied:

(i) $G$ acts on $C$ by automorphism;

(ii) the inductively defined sequence

$$
\Gamma_{1} C=C_{1} \supset \cdots \supset \Gamma_{n+1} C=\left\{g c-c, g \in G, c \in \Gamma_{n} C\right\}
$$

vanishes for some $n \geq 1(\{\cdots\}$ denotes the $Z G$-submodule in $C$ generated by $g c-c)$.

[TH]-Theorem (J.-C. Thomas). Let (*) be a Serre fibration satisfying the previous restrictions. Suppose that $\pi_{1}(B)$ acts nilpotently on $H^{j}(F), j \geq 1$. Then there exists a model $\left(A^{*}(E), d\right)$ which is a twisted tensor product

$$
\left(A^{*}(E), d\right)=\left(\mathcal{M}_{B}, d_{B}\right) \otimes_{\tau}\left(\mathcal{M}_{F}, d_{F}\right)
$$

of the minimal models $\left(\mathcal{M}_{B}, d_{B}^{*}\right)$ and $\left(\mathcal{M}_{F} d_{F}\right)$ of $B$ and $F$ respectively. The twisting $r$ is of the form (3).

Remark. We prefer to write the twisting $r$ in the form [TeS].

In the sequel we denote the Lie algebra of a Lie group $G$ by $L(G)$. The dual of the vector space $X$ is denoted by $X^{*}$. 
Following [BG2] we consider as solvmanifolds only homogeneous spaces $G / \Gamma$, where $G$ is a solvable simply connected Lie group and $\Gamma$ is a lattice in $G$, that is a discrete co-compact subgroup (see[Au, $\mathrm{M}$ ] for general theory of solvmanifolds).

We introduce nilmanifolds as homogeneous spaces $N / \Gamma$, where $N$ is a simply connected nilpotent Lie group and $\Gamma$ a lattice in $N$.

To compare our results with [BG2,FLS], we recall the definition of a completely solvable Lie group: a Lie group $G$ is completely solvable, if all endomorphisms $a d V: L(G) \rightarrow L(G), V \in L(G)$ possess only real eigenvalues. Of course, there are many solvable Lie groups which are not completely solvable (see e.g. [Au], [VGS]).

To prove the main result of this paper we need the following fact, which is well-known and can be found in the explicit form in $\mid \mathrm{O}]$ :

The minimal model of any compact nilmanifold $N / \Gamma$ is of the form

$$
\left(\mathcal{M}_{N / \Gamma}, d\right) \simeq\left(\Lambda L(N)^{*}, \delta\right)
$$

where $\delta$ is a standard derivation determining the cohomology of $L(N)$ :

$$
\delta \alpha\left(X_{0}, \cdots X_{k}\right)=\sum_{i<j}(-1)^{i+j-1} \alpha\left(\left[X_{i}, X_{j}\right], X_{0}, \cdots, \widehat{X}_{i}, \widehat{X}_{j}, \cdots X_{k}\right),
$$

$X_{0}, \cdots X_{k} \in L(N), \alpha \in \Lambda^{k} L(N)^{*}$.

In Section 4 we use the notion of the higher-order Massey product, referring to $[\mathrm{K}]$ for the definition.

A free graded algebra over a graded vector space $X$ is denoted by $\Lambda X$, the degree of an element $x \in X$ is denoted by $\operatorname{deg}(x)$.

Analyzing examples in the last section of the paper, we refer to the Kodaira classification of compact complex surfaces [BPV] and [FM]. We use the Kodaira classification table in the form [BPV] (p.188). All definitions necessary to understand this classification are also contained in [BPV]. We denote the geometric genus of a surface by the symbol $\pi_{g}$ and the irregularity number by $q$.

\section{Proof of the theorem and corollary}

Let $(\Lambda X \otimes \Lambda Y, d)$ satisfy the conditions of the Theorem. In the sequel we assume $X$ and $Y$ to be finite dimensional and fix the bases $x_{1}, \cdots x_{k}, y_{1}, \cdots, y_{l}$ of $X$ and $Y$ respectively. We begin with 
Lemma 1. Let $(\Lambda X \oplus \Lambda Y, d)$ satisfy the condition (ii) of the Theorem. Suppose that $(\Lambda X \oplus \Lambda Y, d)$ is oriented. Then, necessarily, the derivation $d$ is of the form

$$
d y_{j}=x^{(j)}+y_{s_{j}} t_{j}+\sum_{s \neq j} \tilde{x}_{s} y_{s}+\bar{x}_{j} y_{j}, j=1, \cdots l
$$

where

$$
\begin{gathered}
\sum_{j=1}^{l} \bar{x}_{j}=0, \\
x^{(j)} \in \Lambda^{2} X, y_{s_{j} t_{j}} \in \Lambda^{2} Y, \operatorname{deg}\left(\tilde{x}_{s}\right)=1, \operatorname{deg}\left(y_{s}\right)=1, \operatorname{deg}\left(\bar{x}_{j}\right)=1
\end{gathered}
$$

Proof. Since $H^{n}(\Lambda X \otimes \Lambda Y, d) \neq 0$ and since $(\Lambda X \otimes \Lambda Y)$ is the exterior algebra,

$$
\operatorname{dim} H^{n}(\Lambda X \otimes \Lambda Y)=\operatorname{dim} Z^{n}(\Lambda X \otimes \Lambda Y)=\operatorname{dim} \Lambda^{n}(X \oplus Y)=1 .
$$

Suppose that $\Lambda^{n-1}(X \oplus Y) \neq Z^{n-1}(\Lambda(X \oplus Y))$, then there exists $v \in$ $\Lambda^{n-1}(X \oplus Y), d v \neq 0, d v \in \Lambda^{n}(X \oplus Y)$. By the previous remark $d v=\alpha u$, where $u$ is a generator in $\Lambda^{n}(X \oplus Y)$, corresponding to the non-zero cohomology class, which is a contradiction.

Thus

$$
\Lambda^{n-1}(X \oplus Y)=\Lambda^{k-1, l}+\Lambda^{k, l-1}=Z^{n-1}(X \oplus Y)
$$

Therefore

$$
u_{i}=x_{1} \cdots \widehat{x}_{i} \cdots x_{k} y_{1} \cdots y_{l} \in \Lambda^{k-1, l}, i=1, \cdots, k
$$

are cocycles (here and in the sequel $\widehat{x}_{i}$ denotes the absence of $x_{i}$ ). Calculating $d u_{i}$ explicitly one obtains

$$
\begin{gathered}
d u_{i}=x_{1} \cdots \widehat{x}_{i} \cdots x_{k}\left((-1)^{j-1} \sum_{j=1}^{l} y_{1} \cdots y_{j-1} d y_{j} y_{j+1} \cdots y_{l}\right)= \\
=x_{1} \cdots \widehat{x}_{i} \cdots x_{k}\left\{(-1)^{j-1} \sum_{j=1}^{l} y_{1} \cdots y_{j-1}\left(x^{(j)}+y_{s_{j} t_{j}}+\sum_{s \neq j} \widetilde{x}_{s} y_{s}+\bar{x}_{s} y_{s}+\bar{x}_{j} y_{j}\right)\right. \\
\left.y_{j+1} \cdots y_{l}\right\}=
\end{gathered}
$$




$$
\begin{gathered}
=\sum_{j=1}^{t}(-1)^{j-1}\left\{x_{1} \cdots \widehat{x}_{i} \cdots x_{k} y_{1} \cdots y_{j-1} x^{(j)} y_{j+1} \cdots y_{l}+x_{1} \cdots\right. \\
\widehat{x}_{i} \cdots x_{k} y_{1} \cdots y_{j-1} y_{s} t_{j} y_{j+1} \cdots y_{l}+x_{1} \cdots \widehat{x}_{i} \cdots x_{k} y_{1} \cdots y_{j-1}\left(\sum_{s \neq j} \tilde{x}_{s} y_{s}\right) y_{j+1} \cdots \\
\left.y_{t}+x_{1} \cdots \widehat{x}_{i} \cdots x_{k} y_{1} \cdots y_{j-1} \bar{x}_{j} y_{j} y_{j-1} \cdots y_{l}\right\}=0
\end{gathered}
$$

Observe that

$$
\begin{gathered}
x_{1} \cdots \widehat{x}_{i} \cdots x_{k} y_{1} \cdots y_{j-1} x^{(j)} y_{j+1} \cdots y_{l} \in \Lambda^{k+1, l}=\{0\} \\
x_{1} \cdots x_{i} \cdots x_{k} y_{1} \cdots y_{j-1} y_{s_{j} t_{j}} y_{j+1} \cdots y_{l} \in \Lambda^{k-1, l+1}=\{0\} \\
x_{1} \cdots \widehat{x}_{i} \cdots x_{k} y_{1} \cdots y_{j-1}\left(\sum_{s \neq j} \tilde{x}_{s} y_{s}\right) y_{j+1} \cdots y_{l}=0 .
\end{gathered}
$$

The first two equalities are obvious, the third follows, since $s \in\{1, \cdots, j-1, j+1, \cdots, l\}$. Finally

$$
x_{1} \cdots \widehat{x}_{i} \cdots x_{k}\left(\sum_{j=1}^{l} \bar{x}_{j}\right) y_{1} \cdots y_{j-1} y_{j} y_{j+1} \cdots y_{l}=0
$$

which means that

$$
\sum_{j=1}^{l} \bar{x}_{j} \in A n n_{\Lambda^{1,0}}\left(x_{1} \cdots \widehat{x}_{i} \cdots x_{k}\right)
$$

Since the above argument is valid for all $i$,

$$
\sum_{j=1}^{l} \bar{x}_{j} \in \mathrm{n}_{i=1}^{k} A n n_{\Lambda^{1,0}}\left(x_{1} \cdots \widehat{x}_{i} \cdots x_{k}\right)
$$

Since the sum of $\bar{x}_{j}$ is of degree $1,(3)$ follows. Lemma 1 is proved.

Lemma 2. Under the conditions of Lemma 1 the inclusion holds:

$$
d\left(\Lambda^{k-2, l}\right) \subset \Lambda^{k, l-1}
$$


Proof. Take the element $y_{1} \cdots y_{l}$. Then

$$
\begin{gathered}
d\left(y_{1} \cdots y_{l}\right)=\sum_{i=1}^{l}(-1)^{i-1} y_{1} \cdots d y_{i} \cdots y_{l}= \\
=\sum_{i=1}^{l}(-1)^{i-1} y_{1} \cdots y_{i-1}\left(x^{(i)}+y_{s_{i} t_{i}}+\sum_{s \neq i} \tilde{s}_{s} y_{s}+\bar{x}_{i} y_{i}\right) y_{i+1} \cdots y_{l}= \\
=\sum_{i=1}^{l}(-1)^{i-1} y_{1} \cdots y_{i-1} \bar{x}^{(i)} y_{i+1} \cdots y_{l}+\sum_{i=1}^{l}(-1)^{i-1} y_{1} \cdots y_{i-1} \bar{x}_{i} y_{i} y_{i+1} \cdots y_{l}= \\
=\sum_{i=1}^{l}(-1)^{i-1} y_{1} \cdots y_{i-1} x^{(i)} y_{i+1} \cdots y_{l} \in \Lambda^{2, l},
\end{gathered}
$$

because

$$
\begin{gathered}
y_{1} \cdots y_{i+1} y_{s_{i} t_{i}} y_{i+1} \cdots y_{l} \in \Lambda^{0, l+1}=\{0\} \\
y_{1} \cdots y_{i-1}\left(\sum_{s \neq i} \tilde{x}_{s} y_{s}\right) y_{i+1} \cdots y_{t}=0
\end{gathered}
$$

and

$$
\sum_{i=1}^{l}(-1)^{2(i-1)} \bar{x}_{i} y_{1} \cdots y_{i-1} y_{i} y_{i+1} \cdots y_{l}=\left(\sum_{i=1}^{l} \bar{x}_{i}\right) y_{1} \cdots y_{l}=0
$$

from Lemma 1. Lemma 2 is proved.

Lemma 3. Let $(\Lambda X \otimes \Lambda Y, d)$ satisfy the conditions of Lemma 7. Assume in addition that the Poincaré duality holds. If

$$
H^{1}(\Lambda X \otimes \Lambda Y, d)=\Lambda^{1,0},
$$

then

$$
H^{n-1}(\Lambda X \otimes \Lambda Y, d)=\Lambda^{k-1, l} .
$$

Proof. From the Poincaré duality

$$
\operatorname{dim} H^{n-1}(\Lambda X \times \Lambda Y)=k .
$$

From (4)

$$
Z^{n-1}(\Lambda X \otimes \Lambda Y)=\Lambda^{k-1, l}+\Lambda^{k, l-1}
$$


and by comparing the degrees (the calculation is straight forward, although it is necessary to use the minimality of $(\Lambda Y, \delta)$ which guarrantees that $y_{t_{j} s_{j}}$ do not contain expressions with $y_{j}$ ):

$$
d\left(\Lambda^{k-1, l-1} \subset \Lambda^{k, l-1}, d\left(\Lambda^{k, l-2}\right) \subset \Lambda^{k, l-1}\right.
$$

From Lemma 2 (Formula (5))

$$
d\left(\Lambda^{k-2, l}\right) \subset \Lambda^{k, l-1}
$$

and therefore, the subspace of coboundaries $B^{n-1} \subset \Lambda^{k, l-1}$. Thus,

$$
\operatorname{dim} H^{n-1}(\Lambda X \otimes \Lambda Y)=\operatorname{dim}\left(\Lambda^{k-1, l}+\Lambda^{k, l-1}\right)-\operatorname{dim} B^{n-1}=k
$$

Therefore, since $\operatorname{dim} \Lambda^{k, l-1}=l, \operatorname{dim} \Lambda^{k-1, l}=k$, the assumption $B^{n-1} \neq$ $\Lambda^{k, l-1}$ would imply

$$
\operatorname{dim} H^{n-1}=\operatorname{dim} Z^{n-1}-\operatorname{dim} B^{n-1}=k+l-\operatorname{dim} B^{n-1}>k .
$$

Thus

$$
B^{n-1}=\Lambda^{k, l-1}
$$

and Lemma 3 is proved.

\section{Proof of the Theorem}

We begin the proof showing that there exists a graded differential algebra which is a model for $G / \Gamma$ and which satisfies the condition (i) of the Theorem. Then we prove that this algebra possesses algebraic properties (ii)-(v) stated in the Theorem.

Let $N$ be the nilradical of $G$. From the Mostow theorem [M], $N \subset \Gamma$ is a lattice in $N$. The latter fact is equivalent to the closeness of $N \Gamma$ in $G$. Therefore, one can construct the "double fibration"

$$
\Gamma N=N / \cap \Gamma \rightarrow G / \Gamma \rightarrow G / \Gamma N .
$$

Since $G / \Gamma=G \times{ }_{N}(N / N \cap \Gamma)$, the fiber of the locally trivial bundle above possesses the path-connected structural group $N$. From the Serre theorem $[\mathrm{Se}], H^{*}(N / N \cap \Gamma)$ is a trivial $\pi_{1}(G / N \cap \Gamma)$-module. Since all cohomology algebras here are of a finite type, the Thomas theorem (Section 2) is applicable and the rational model $A^{*}(G / \Gamma)$ of $G / \Gamma$ is a twisted tensor product

$$
\mathcal{M}_{G / \Gamma N} \otimes_{\tau} \mathcal{M}_{N / N \cap \Gamma}
$$


It is well known that $G / \Gamma N$ is a torus and since $\Gamma \cap N$ is a lattice, the fiber of the considered fibration is a compact nilmanifold. Finally,

$$
\mathcal{M}_{G / \Gamma N}=(\Lambda X, d=0), \mathcal{M}_{N / N \cap F}=\left(\Lambda L(N)^{*}, \delta\right)
$$

(see Section 2) and

$$
\left(A^{*}(G / \Gamma), d\right)=(\Lambda X, 0) \otimes_{\tau}(\Lambda Y, \delta),
$$

where (i) and (ii) are satisfied, $Y=L(N)^{*}$. Suppose that $(\Lambda X \otimes \Lambda Y, d)$ satisfies the conditions of Lemma 1 and $d y_{j}$ are linearly independent. Then (6) holds, since $d \mid X=0$ and $\left.d\right|_{Y}$ is one-to-one. If some $d y_{j}$ are linearly dependent, from the obvious base change one can assume that for the new basic vectors $\bar{y}_{j}, d \bar{y}_{j}=0$ and thus $\bar{y}_{j} \in X$. Therefore one can assume the linear independence of $d y_{j}$ without loss of generality and (7) follows. Now, the condition (iii) follows from Lemma 3.

To prove (iv)-(v), take $y^{*} \in Y^{*}$ and observe that

$$
\left(i\left(y^{*}\right) \omega\right) \omega^{m-1}=\frac{1}{m} i\left(y^{*}\right) \omega^{m} \in \Lambda^{k, l-1},
$$

since $\omega^{m} \in \Lambda^{n}=\Lambda^{k+l}$. From (8) $i\left(y^{*}\right) \omega^{m}$ is a coboundary. Therefore,

$$
i\left(y^{*}\right) \omega \notin \Lambda^{1,0}
$$

because otherwise the hard Lefschetz condition and Lemma 3 would give a contradiction. Thus there exists $\bar{y}^{*} \in Y^{*}$ such that $\omega\left(y^{*}, \bar{y}^{*}\right) \neq 0$ and therefore $\left.\omega\right|_{Y^{*} \times} Y^{*}$ is non-degenerate. Observe that using nondegeneracy, one can choose $X^{*}$ to be $\omega$-orthogonal to $Y^{*}$. Using the appropriate identifications of exterior algebra elements and alternating forms, one obtains the form of the Lefschetz element, that is (iv) and, as a consequence, $(v)$. The Theorem is proved.

\section{Proof of the Corollary}

Analyzing the proof of the Theorem one can notice that conditions (iii)-(v) are valid for any twisted tensor product satisfying the Lefschetz conditions and (ii). Following [BG2] we assign to $G / \Gamma$ a model $\bar{A}^{*}(G / \Gamma)$ which possesses properties (ii)-(v) of $A^{*}(G / \Gamma)$, although this new model in general differs from the latter. We can observe that for completely solvable Lie groups

$$
H^{*}(G / \Gamma) \simeq H^{*}(L(G))=H^{*}\left(\Lambda L(G)^{*}, \delta\right)
$$


from Hattori's theorem [H]. Since

$$
\left(\Lambda L(G)^{*}, \delta\right) \simeq\left(\Omega_{D R}^{i n v}(G), d\right),
$$

the cohomology class of a Kahlerian form has a representative $\omega \in$ $\left(\Lambda^{2} L(G)^{*}, \delta\right)$. Since $\omega^{m} \neq 0$ and $\omega$ is an alternarive 2-form on $L(G)$, it is non degenerate. Therefore, $\left(\Lambda L(G)^{*}, \delta\right)$ can be taken as a model of $G / \Gamma$.

Take $\mathcal{N}=[L(G), L(G)]$ and decompose $L(G)^{*}$ as a vector space sum

$$
L(G)^{*}=\mathcal{A}^{*} \oplus \mathcal{N}^{*},
$$

where

$$
\mathcal{A}^{*}=\left\{\theta \in L(G)^{*} \mid \theta(\mathcal{N})=0\right\}, \mathcal{N}^{*}=\left\{\alpha \in L(G)^{*} \mid \alpha(\mathcal{A})=0\right\}
$$

(here $\mathcal{A}$ is an arbitrary complement to $\mathcal{N}$ ). Now, taking into account the equalities (the first follows from (2))

$$
\delta \beta(U, V)=-\beta([X, Y]), \mathcal{N}=[L(G), L(G)]
$$

we have

$$
\delta(\mathcal{A})=0
$$

Since $\mathcal{N} \subset$ in the nilradical of $L(G)$, the ideal $\mathcal{N}$ is nilpotent and therefore

$$
\left(\Lambda \mathcal{N}^{*},\left.\delta\right|_{\mathcal{N}^{*}}\right)
$$

is a minimal graded differential algebra. Therefore,

$$
\left(\bar{A}^{*}(G / \Gamma)=\left(\Lambda L(G)^{*}, \delta\right)=\left(\Lambda \mathcal{A}^{*}, \delta=0\right) \otimes_{\tau}\left(\Lambda \mathcal{N}^{*},\left.\delta\right|_{\mathcal{N}^{*}}\right)\right.
$$

(that is, the model for $G / \Gamma$ can be represented as a twisted tensor product satisfying the condition (ii) of the Theorem, the Lefschetz condition and the conditions of Lemmata 1-3 (the latter is guaranteed by the minimality of the second term in the twisted tensor product). Finally, $\left(\bar{A}^{*}(G / \Gamma), d\right)$ satisfies the properties (i)-(v) of the Theorem and as a consequence, the properties (ii), (iv) and (v) of the Corollary.

Remark. These properties were proved in [BG2] directly for the particular case of $\left(\Lambda L(G)^{*}, \delta\right)$ with completely solvable $G$. 
To finish the proof it is enough to repeat the argument in [BG2] (parts (i), (iii) and (vi). Here, of course, the proof is based on the particular choice of $\left(\bar{A}^{*}(G / \Gamma), d\right)$. For example, the commutativity of $\mathcal{A}$ follows from (v) of the Theorem and the formula

$0=\delta \omega(A, B, V)=-\omega([A, B], V)+\omega([A, V], B)-\omega([B, V], A), A, B \in \mathcal{A}, V \in \mathcal{N}$

(see the proof in [BG2] for details). The Corollary is proved.

\section{Examples}

It is rather easy to produce examples of solvmanifolds which are not completely solvable. For instance, any 4-dimensional real solvable Lie group possessing lattices is either nilpotent, or is of one of two forms below:

a) $G=R \times{ }_{\varphi} R^{3}$, where $\varphi: R \rightarrow G L_{3}(R)$ is a homomorphism satisfying the condition $\varphi(1) \in G L_{3}(Z)$, or

b) $G=R \times_{\varphi} N_{3}$, where $N_{3}$ is a maximal unipotent subgroup in $S L_{3}(R)$ and $\varphi: R \rightarrow A u t\left(N_{3}\right)$ is a homomorphism such that $\varphi(1)$ preserves the subgroup $N_{3}(Q)$ in $S L_{3}(Q)$ of matrices with rational entries in $N_{3}$ (see [VGS] and [Au]).

Following this general description, we produce the following example.

Example 1. Let $G$ and $\varphi$ be as in a) above and define $\phi$ by the formula

$$
\varphi(t)=\left(\begin{array}{ccc}
\cos t 5 \pi & \sin t 5 \pi & 0 \\
-\sin t 5 \pi & \cos t 5 \pi & 0 \\
0 & 0 & 1
\end{array}\right)
$$

Then there exists a lattice $\Gamma$ in $G$ such that $G / \Gamma$ is a compact symplectic solvmanifold which is not diffeomorphic to a manifold. This solvmanifold is not completely solvable and has no Kählerian structure.

To prove this assertion, observe that the Lie algebra of $G$ is of the form

$$
L(G)=S p a n(A, X, Y, Z),[A, X]=-Y,[A, Y]=X,[A, Z]=0
$$

and all other brackets are zero. Since

$$
L(G)=\mathcal{A} \times_{\phi_{*}} \mathcal{B}, \mathcal{A}=\operatorname{Span}(A), \mathcal{B}=\operatorname{Span}(X, Y, Z)
$$


where $\mathcal{A}$ and $\mathcal{B}$ are abelian, $L(G)$ is solvable. It is non-nilpotent, since $\operatorname{ad}(A)^{n}(X) \neq 0$ for any $n$. Obviously $\operatorname{ad}(A)$ has imaginary eigenvalues and $G$ is not completely solvable. Since $\phi(1)$ has integer entries, $G$ possesses a lattice. If we generate the lattice in $R$, say, by $\frac{1}{5}$, we obtain a lattice $\Gamma$ which is a non-nilpotent group extending $Z^{4}$ by a group $Z / 5 Z$. And therefore $G / \Gamma$ is not diffeomorphic to a nilmanifold.

Observe that $G / \Gamma$ is symplectic. To show this, consider the complex $\left(\Lambda L(G)^{*}, \delta\right)$. From Section 2 one can write

$$
\begin{gathered}
\left(\Lambda L(G)^{*}, \delta\right)=(\Lambda(a, x, y, z), \delta), \\
\delta(a)=0, \delta(x)=-a y, \delta(y)=a x, \delta(z)=0 .
\end{gathered}
$$

One can check that

$$
\omega=a z+x y
$$

is a symplectic form on $G / \Gamma$ (one identifies $\Lambda L(G)^{*}$ with left-invariant forms on $G$ and projects this forms on $G / \Gamma$ ).

We cannot use the Hattori theorem to prove the non-existence of a Kählerian structure, since $G / \Gamma$ is not completely solvable. To prove the latter assertion we appeal to the Kodaira classification of complex surfaces. Assuming $G / \Gamma$ to be Kählerian and hence complex one performs the following argument. Since $G / \Gamma$ is parallelizable, its Euler characteristic and the first Pointriagin number vanish, which implies the vanishing of the Chern classes $c_{1}$ and $c_{2}$. Therefore, the geometric genus $p_{g}(G / \Gamma)=0$. Then the Kodaira classification of complex surfaces implies $G / \Gamma$ to be algebraic [BPV]. One can notice that if $\Gamma=A \times \times_{\varphi} B$ is a semidirect product of two abelian groups determined by the homomorphism $\varphi: A \rightarrow A u t(B)$, then $\Gamma /[\Gamma, \Gamma]$ consists of elements of the form

$$
\left(e, b \cdot \varphi(a)\left(b_{1}\right) \cdot \varphi\left(a_{1}\right)\left(b^{-1}\right) \cdot b_{1}^{-1}\right)
$$

Calculating this expression for $\Gamma$ and $\varphi$ determining our particular solvmanifold, one can check directly that

$$
\operatorname{rank}(\Gamma /[\Gamma, \Gamma])=2,
$$

which implies that $b_{1}(G / \Gamma)=2$. From the Kodaira classification table [BPV] (p. 188) $G / \Gamma$ cannot be a surface of general type. Then, since the geometric genus vanishes and the second Betti number is 2, one obtains 
$q(G / \Gamma)=h^{1}\left(\mathcal{O}_{G / \Gamma}\right)=1$. Using the same argument as in [FM] (p. 30), one eliminates ruled surfaces. The only possibility remains to consider is that of hyperelliptic surfaces. In the latter case,

$$
G / \Gamma=(B \times F) / H,
$$

where $B$ and $F$ are elliptic curves and $H$ is a finite group acting freely on $B \times F$ and the genera $g(B), g(F) \geq 1$. Since $H$ is always of the form $H=Z / m Z, m=2,3,4,6$ [FM], the possible fundamental group of $(B \times F) / H$ is an extension of $Z^{4}$ by $Z / m Z$ with $m=2,3,4,6$ and from our construction

$$
\pi_{1}(G / \Gamma) \neq \pi_{1}((B \times F) / H)
$$

which implies the proof.

\section{Example 2.}

Proposition 1. Let $M=G / \Gamma$ be a compact 4-dimensional solvmanifold. If $M$ admits a Kähler structure, then $M$ has the rational homotopy type either of a torus $T^{4}$, or of $S^{2} \times T^{2}$.

Proof. Assuming $M$ to be Kähler and hence complex, we appeal to the Kodaira classification of complex surfaces. From the table in [BPV], either $b_{1}(M)=4$, or $b_{1}(M)=2$, since $b_{1}(M)=1$ and $b_{1}(M)=3$ are eliminated by the Kähler condition, and $b_{1}(M)=0$ cannot be realized by a compact solvmanifold of the type a) or b) above.

Now, apply the Theorem proved in this paper. Applying the proof of it to the Lie groups $G$ of the form a) b), one obtains

$$
\left(A^{*}(G / \Gamma), d\right)=(\Lambda(a, b, x, y), d),
$$

and from parts (iii) and (v) it follows that either

$$
d(a)=d(b)=d(x)=d(y)=0,
$$

or

$$
d(a)=d(b)=0, d(x) \neq 0, d(y) \neq 0 .
$$

We claim now, that part (iv) shows that the differential $d$ in $\left(A^{*}(G / \Gamma), d\right)$ up to isomorphism is either of the form

$$
d(a)=d(b)=0, d(x)=-a y, d(y)=a x,
$$


or

$$
d(a)=d(b)=0, d(x)=a x, d(y)=-a y .
$$

To prove the latter assertion, it is enough to make on obvious variable change, taking into consideration the identities

$$
d^{2}=0, \text { and } d(x y)=0
$$

(the latter follows from (iv), since the Lefschetz element is necessarily of the form $a b+x y$. Calculations are straightforward, therefore we won't reproduce them. Note only, that it is convenient to rewrite $d$, say, in the form

$$
d(x)=a_{11} x+a_{12} y, d(y)=a_{21} x+a_{22} y, a_{i j} \in \operatorname{Span}(a, b)
$$

and write explicitly the conditions on $a_{i j}$ following from (9). Then the appropriate change of variables can be seen easily.

Now, it is enough to notice that the cohomology algebra $H^{*}(\Lambda(a, b, x, y), d)$ in both cases is generated by elements $a, b, a b, x y, a x y, b x y, a b x y$. Then some straghtforward computation shows that $H^{*}(\Lambda(a, b, x, y), d)$ has the minimal model given by the formula

$$
\begin{gathered}
\left(\mathcal{M}, d_{1}\right)=\left(\Lambda(a, b,) \otimes R\left[X_{1}\right] \otimes \Lambda\left(y_{1}\right), d_{1}\right), \\
d(a)=d(b)=d\left(X_{1}\right)=0, d\left(y_{1}\right)=X_{1}^{2}, \operatorname{deg}\left(X_{1}\right)=2, \operatorname{deg}\left(y_{1}\right)=3 .
\end{gathered}
$$

Since we assumed $G / \Gamma$ to be Kählerian, its minimal model is a formal consequence of the cohomology algebra $H^{*}(G / \Gamma)$ [DGMS]. Then, the uniqueness theorem for the minimal model (which does not depend on the nilpotency condition) implies.

$$
\mathcal{M} \simeq \mathcal{M}_{G / \Gamma} \simeq \mathcal{M}_{T^{2} \times S^{2}}
$$

The proposition is proved.

\section{Example 3.}

Many more examples can be constructed. We restrict ourselves to indicating one more example in dimension 8 which is a modification of an example in [FLS], but which also cannot be handled by the techniques of [FLS]. 
Proposition 2. Let $L(G)$ ba a Lie algebra defined as follows

$$
\begin{gathered}
L(G)=\operatorname{Span}\left(A, B, X_{1}, X_{2}, X_{3}, X_{4}, X_{5}, X_{6}\right), \\
{\left[A, X_{1}\right]=X_{1},\left[A, X_{2}\right]=-X_{2},\left[A, X_{3}\right]=X_{3},\left[A, X_{4}\right]=-X_{4}} \\
{\left[A, X_{5}\right]=\alpha X_{6},\left[A, X_{6}\right]=-\alpha X_{5},\left[B, X_{3}\right]=X_{1},\left[B, X_{4}\right]=X_{2}, \alpha \in R}
\end{gathered}
$$

(the other brackets are assumed to be zero).

Let $G$ be a simply-connected Lie group corresponding to $L(G)$. Then:

(i) $G$ is a solvable non-nilpotent and not completely solvable Lie group;

(ii) $G$ contains a lattice $\Gamma$ for some particular $\alpha$;

(iii) the homogeneous space $G / \Gamma$ is a compact symplectic solvmanifold with no Kähler structure

The proof of this proposition is given in $[\mathrm{Tr}]$ and is based on the quite different argument combined with a long calculation using quadruple Massey products [K]. We omit it here.

Remark. Our examples are analogous to Example 1 in [BG2] and Theorems $1-3$ in [FG] when $G / \Gamma$ is not completely solvable.

Examples presented here give some evidence for the following

Conjecture. A compact solvmanifold $G / \Gamma$ of $(R)-$ type is Kählerian if and only if it is a homotopy torus.

By definition [VGS], a solvmanifold $G / \Gamma$ is of $(R)$-type, if for any $V \in L(G)$ all linear operators adV $L(G) \rightarrow L(G)$ have only imaginary eigenvalues.

Acknowledgement. This work was supported by the Polish KBN. The author is grateful to Martin Saralegi for the opportunity of reading the preprint [FLS] before its publication and to the referee for valuable suggestions.

\section{References}

[Ab] E. Abbena, An example of an almost Kähler manifold which is not Kählerian, Boll. Un. Mat. Ital (6) 3-A (1984), 383-392. 
[Au] L. Auslander, An exposition of the structure of solvmanifolds, Bull. Amer. Math. Soc. 79 (1973), 273-285.

[BG1] C. Benson, C. S. Gordon, Kähler and symplectic structures on nilmanifolds, Topology 27 (1988), 513-518.

[BG2] C. Benson, C. S. Gordon, Kähler structures on compact solvmanifolds, Proc. Amer. Math. Soc. 108 (1990), 971-980.

[BPV] W. Barth, C. Peters, A. Van den Ven, Compact Complex Surfaces, Springer, Berlin, 1984.

[CFG] L. A. Cordero, M. Fernández, A. Gray, Symplectic manifolds with no Kähler structure, Topology 25 (1986), 375-380.

[DGMS] P. Deligne, P. Griffiths, J. Morgan, D. Sullivan, Real homotopy theory of Kähler manifolds, Inventiones Math. 29 (1975), 245-274.

[Gmf] R. Gompf, Some new examples of symplectic 4-manifolds, preprint MPI/92-47 (1992).

[H] A. Hattori, Spectral sequence in the de Rham cohomology of fibre bundles, J. Fac. Sci. Univ. Tokyo, Sect. 18 (1960), 289-331.

[Ha] S. Halperin, Lectures on Minimal Models, Hermann, Paris, 1982.

[Le] D. Lehmann, Théorie homotopique des formes differentielles (d'après D. Sullivan), Societé Math. de France, Asterisque 45 (1977).

[FG] M. Fernández, A. Gray, Compact symplectic solvmanifolds not admitting complex structures, Geom. Dedic. 34 (1990), 295-299.

[FLS] M. Fernández, M. de León, M. Saralegi, A six-dimensional compact symplectic solvmanifold without Kähler structures, preprint (1992).

[FM] R. Friedman, J. Morgan, Smooth Four-Manifolds and Complex Surfaces, Springer, Berlin, 1994.

[Has] K. Hasegawa, Minimal models of nilmanifolds, Proc. Amer. Math. Soc. 106 (1989), 65-71. 
[K] D. Kraines, Massey higher products, Trans. Amer. Math. Soc. 124 (1996), 431-449.

[LuO] G. Lupton, J. Oprea, Symplectic manifolds and formality, J. Pure and Appl. Algebra 91 (1994), 193-207.

[M] G. D. Mostow, Factor spaces of solvable groups, Annals of Math. 60 (1954), 1-27.

[McD] D. McDuff, Examples of symplectic simply conneeted manifolds with no Kähler structure, J. Diff. Geom. 20 (1984), 267-277.

[O] J. Oprea, The category of nilmanifolds, Enseign. Math. 38 (1992), $27-40$.

[R] M. Raghunatan, Discrete Subgroups of Lie Groups, Springer, Berlin, 1972.

[Se] J.-P. Serre, Homologie singulière des espaces fibrès, Annals of Math. 54 (1951), 425-505.

[Ta] D. Tanré, Homotopie rationelle: models de Chen, Quillen, Sullivan, Springer Berlin, 1988.

[TeS] M. Tezuka, T. Shiga, Rational fibrations, homogeneous spaces and jacobians, Annales Inst. Fourier (Grenoble) 31 (1987), 81-106.

[Th1] J.-C. Thomas, Homotopie rationelle des fibrès de Serre, Thèse, Université des Sciences et Techniques de Lille I, Lille, 1980.

[Th2] J.-C. Thomas, Rational homotopy of Serre fibrations, Annales Inst. Fourier (Grenoble) 31 (1981), 71-90.

[Tr] A. Tralle, On compact symplectic and Kählerian solvmanifolds which ane not completely solvable, Colloq. Math. (to appear).

[VGS] E. Vinberg, V. Gorbatsevich, O. Shwartzman, Discrete subgroups of Lie groups (in Russian), Itogi Nauki i Techniki. Sovremennyje Problemy Matematiki 21 (1988), 5-115.

Instytut Matematyki

Recibido: 23 de Noviembre de 1995

Uniwersytet Szczeciński

Revisado: 18 de Marzo de 1996

Wielkopolska 15

70-451 Szczecin 3, Poland 